\title{
Syntheses of 2,5-Dimethylcyclopentadienyl Ansa-Zirconocene Complexes and Their Reactivity for Ethylene/Norbornene Copolymerization
}

Eun Sook Cho ${ }^{a}$, Ui Gab Joung ${ }^{a}$, Bun Yeoul Lee ${ }^{a *}$, Hyosun Lee ${ }^{b}$, Young-Whan Park ${ }^{b}$, Choong Hoon Lee $e^{b}$ and Dong Mok Shin ${ }^{c}$

${ }^{\text {a }}$ Department of Molecular Science and Technology, Ajou University, Suwon 443-749

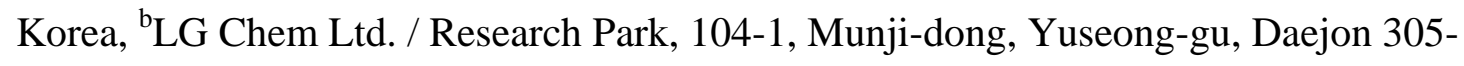

380, Korea, and 'School of Chemistry, Seoul National University, Seoul 151-747,

Korea

bunyeoul@ajou.ac.kr 
${ }^{1} \mathrm{H}$ and ${ }^{13} \mathrm{C}$ NMR spectra of $\left[(\mathrm{Me})(\mathrm{H}) \mathrm{C}\left(1,3-\mathrm{Me}_{2} \mathrm{C}_{5} \mathrm{H}_{2}\right)_{2}\right] \mathrm{Li}_{2}$ (12)
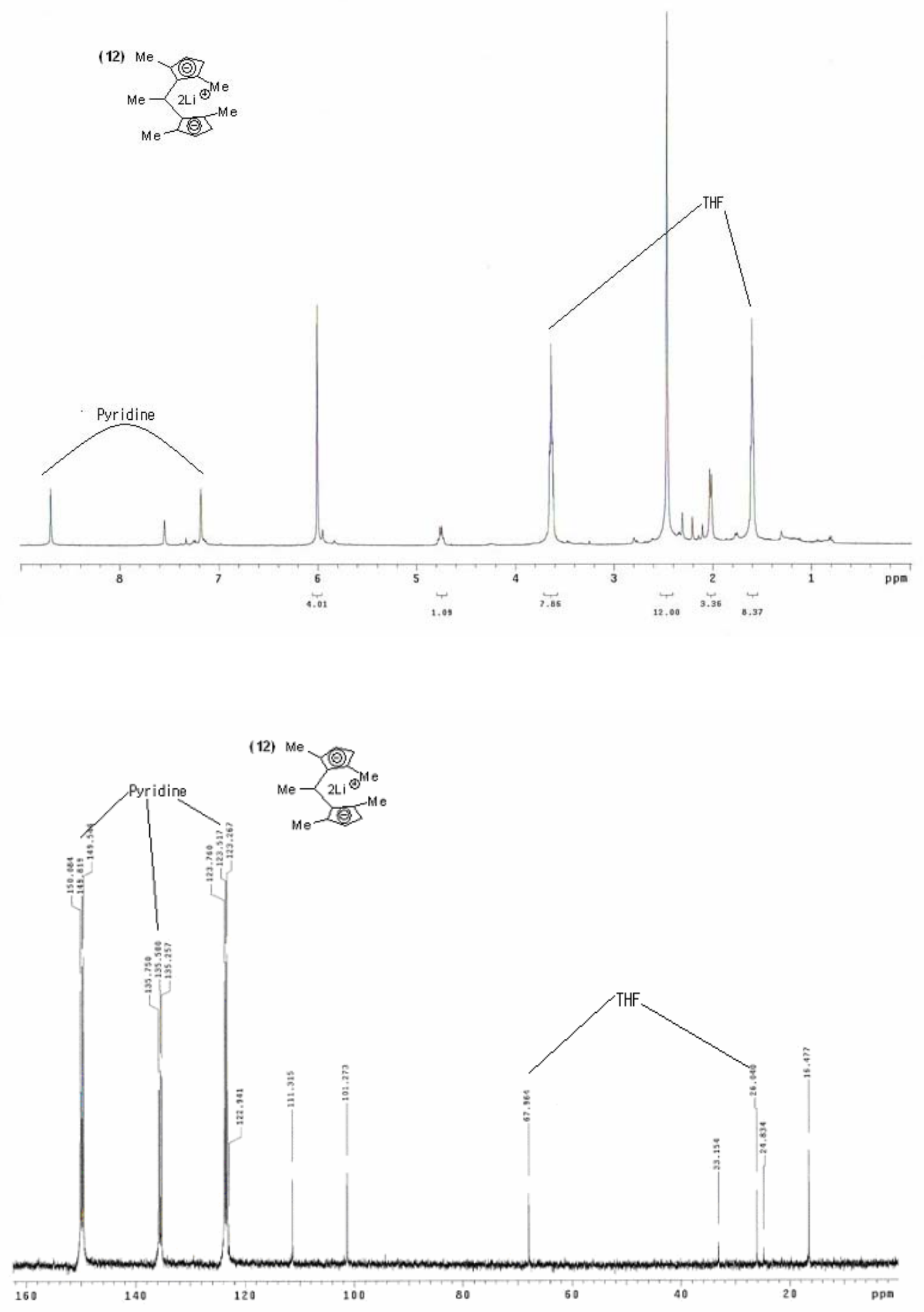


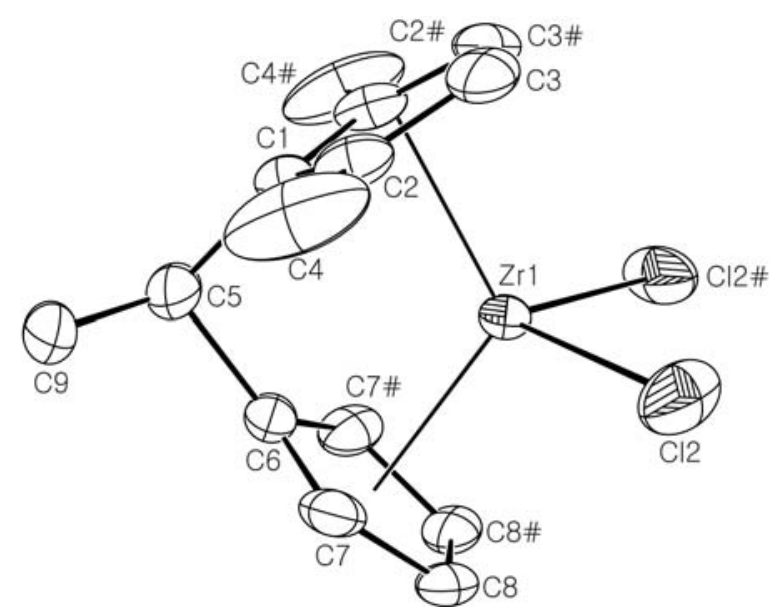

Table 1. Atomic coordinates ( $x$ 10^4) and equivalent isotropic displacement parameters $\left(A^{\wedge} 2 \times 10^{\wedge} 3\right) . U(e q)$ is defined as one third of the trace of the orthogonalized Uij tensor.

\begin{tabular}{lrrrr}
\hline & $\mathrm{x}$ & $\mathrm{y}$ & $\mathrm{z}$ & $\mathrm{U}(\mathrm{eq})$ \\
\hline $\mathrm{Zr}(1)$ & $4328(1)$ & 7500 & $5679(1)$ & $38(1)$ \\
$\mathrm{Cl}(2)$ & $4352(1)$ & $8865(1)$ & $7370(1)$ & $84(1)$ \\
$\mathrm{C}(1)$ & $3276(3)$ & 7500 & $3329(5)$ & $50(1)$ \\
$\mathrm{C}(2)$ & $2854(3)$ & $6679(2)$ & $4108(4)$ & $58(1)$ \\
$\mathrm{C}(3)$ & $2182(3)$ & $7006(3)$ & $5289(4)$ & $74(1)$ \\
$\mathrm{C}(4)$ & $2982(5)$ & $5635(3)$ & $3708(9)$ & $139(3)$ \\
$\mathrm{C}(5)$ & $4311(5)$ & 7500 & $2246(7)$ & $81(2)$ \\
$\mathrm{C}(6)$ & $5361(4)$ & 7500 & $3311(5)$ & $59(1)$ \\
$\mathrm{C}(7)$ & $5780(3)$ & $6685(3)$ & $4088(4)$ & $60(1)$ \\
$\mathrm{C}(8)$ & $6494(2)$ & $7003(2)$ & $5280(4)$ & $52(1)$ \\
$\mathrm{C}(9)$ & $4327(6)$ & $6443(8)$ & $1255(8)$ & $82(3)$ \\
& & & & \\
\hline
\end{tabular}

Table 2. Bond lengths [A] and angles [deg]

\begin{tabular}{llll}
\hline $\operatorname{Zr}(1)-\mathrm{Cl}(2)$ & $2.4353(10)$ & $\operatorname{Zr}(1)-\mathrm{Cl}(2) \# 1$ & $2.4353(10)$ \\
$\operatorname{Zr}(1)-\mathrm{C}(1)$ & $2.436(4)$ & $\operatorname{Zr}(1)-\mathrm{C}(6)$ & $2.439(4)$ \\
$\operatorname{Zr}(1)-\mathrm{C}(7) \# 1$ & $2.455(3)$ & $\operatorname{Zr}(1)-\mathrm{C}(7)$ & $2.455(3)$ \\
$\operatorname{Zr}(1)-\mathrm{C}(2)$ & $2.466(3)$ & $\operatorname{Zr}(1)-C(2) \# 1$ & $2.466(3)$
\end{tabular}




\begin{tabular}{|c|c|c|c|}
\hline $\operatorname{Zr}(1)-C(3)$ & $2.538(3)$ & $\operatorname{Zr}(1)-\mathrm{C}(3) \# 1$ & $2.538(3)$ \\
\hline $\operatorname{Zr}(1)-C(8)$ & $2.560(3)$ & $\operatorname{Zr}(1)-\mathrm{C}(8) \# 1$ & $2.560(3)$ \\
\hline $\mathrm{C}(1)-\mathrm{C}(2) \# 1$ & $1.422(4)$ & $\mathrm{C}(1)-\mathrm{C}(2)$ & $1.422(4)$ \\
\hline$C(1)-C(5)$ & $1.523(7)$ & $\mathrm{C}(2)-\mathrm{C}(3)$ & $1.387(5)$ \\
\hline$C(2)-C(4)$ & $1.500(5)$ & $\mathrm{C}(3)-\mathrm{C}(3) \# 1$ & $1.370(9)$ \\
\hline$C(5)-C(6)$ & $1.527(7)$ & $C(5)-C(9)$ & $1.720(10)$ \\
\hline $\mathrm{C}(5)-\mathrm{C}(9) \# 1$ & $1.720(10)$ & $\mathrm{C}(6)-\mathrm{C}(7) \# 1$ & $1.413(5)$ \\
\hline $\mathrm{C}(6)-\mathrm{C}(7)$ & $1.413(5)$ & $\mathrm{C}(7)-\mathrm{C}(8)$ & $1.416(4)$ \\
\hline $\mathrm{C}(8)-\mathrm{C}(8) \# 1$ & $1.380(7)$ & & \\
\hline $\mathrm{Cl}(2)-\operatorname{Zr}(1)-\mathrm{Cl}(2) \# 1$ & 102.11(7) & $\mathrm{Cl}(2)-\operatorname{Zr}(1)-\mathrm{C}(1)$ & 123.66(5) \\
\hline Cl(2)\#1-Zr(1)-C(1) & $12366(5)$ & $\mathrm{Cl}(2)-\operatorname{Zr}(1)-\mathrm{C}(6)$ & $123.18(5)$ \\
\hline $\mathrm{Cl}(2) \# 1-\operatorname{Zr}(1)-\mathrm{C}(6)$ & $123.18(5)$ & $\mathrm{C}(1)-\operatorname{Zr}(1)-\mathrm{C}(6)$ & $57.59(15)$ \\
\hline $\mathrm{Cl}(2)-\operatorname{Zr}(1)-\mathrm{C}(7) \# 1$ & $90.20(11)$ & $\mathrm{Cl}(2) \# 1-\operatorname{Zr}(1)-\mathrm{C}(7) \# 1$ & $136.04(9)$ \\
\hline C(1)-Zr(1)-C(7)\#1 & 79.12(12) & $\mathrm{C}(6)-\mathrm{Zr}(1)-\mathrm{C}(7) \# 1$ & $33.55(11)$ \\
\hline Cl(2)-Zr(1)-C(7) & 136.04(9) & $\mathrm{Cl}(2) \# 1-\operatorname{Zr}(1)-\mathrm{C}(7)$ & $90.20(11)$ \\
\hline $\mathrm{C}(1)-\mathrm{Zr}(1)-\mathrm{C}(7)$ & $79.12(12)$ & $\mathrm{C}(6)-\mathrm{Zr}(1)-\mathrm{C}(7)$ & 33.55(11) \\
\hline C(7)\#1-Zr(1)-C(7) & $54.8(2)$ & $\mathrm{Cl}(2)-\operatorname{Zr}(1)-\mathrm{C}(2)$ & 136.82(9) \\
\hline Cl(2)\#1-Zr(1)-C(2) & $90.61(9)$ & $\mathrm{C}(1)-\operatorname{Zr}(1)-\mathrm{C}(2)$ & $33.71(10)$ \\
\hline$C(6)-\operatorname{Zr}(1)-C(2)$ & $79.27(13)$ & $\mathrm{C}(7) \# 1-\mathrm{Zr}(1)-\mathrm{C}(2)$ & $108.81(13)$ \\
\hline $\mathrm{C}(7)-\mathrm{Zr}(1)-\mathrm{C}(2)$ & 84.09(13) & $\mathrm{Cl}(2)-\operatorname{Zr}(1)-\mathrm{C}(2) \# 1$ & $90.61(9)$ \\
\hline $\mathrm{Cl}(2) \# 1-\operatorname{Zr}(1)-\mathrm{C}(2) \# 1$ & 136.82(9) & $\mathrm{C}(1)-\operatorname{Zr}(1)-\mathrm{C}(2) \# 1$ & $33.71(10)$ \\
\hline $\mathrm{C}(6)-\operatorname{Zr}(1)-\mathrm{C}(2) \# 1$ & $79.27(13)$ & $\mathrm{C}(7) \# 1-\mathrm{Zr}(1)-\mathrm{C}(2) \# 1$ & 84.09(13) \\
\hline $\mathrm{C}(7)-\mathrm{Zr}(1)-\mathrm{C}(2) \# 1$ & 108.81(13) & $\mathrm{C}(2)-\operatorname{Zr}(1)-\mathrm{C}(2) \# 1$ & $55.03(16)$ \\
\hline Cl(2)-Zr(1)-C(3) & 107.92(11) & $\mathrm{Cl}(2) \# 1-\operatorname{Zr}(1)-\mathrm{C}(3)$ & 83.55(9) \\
\hline $\mathrm{C}(1)-\operatorname{Zr}(1)-\mathrm{C}(3)$ & $5419(13)$ & $\mathrm{C}(6)-\mathrm{Zr}(1)-\mathrm{C}(3)$ & 109.39(14) \\
\hline C(7)\#1-Zr(1)-C(3) & $132.62(12)$ & $\mathrm{C}(7)-\operatorname{Zr}(1)-\mathrm{C}(3)$ & $115.37(13)$ \\
\hline $\mathrm{C}(2)-\operatorname{Zr}(1)-\mathrm{C}(3)$ & $32.15(12)$ & $\mathrm{C}(2) \# 1-\operatorname{Zr}(1)-\mathrm{C}(3)$ & 53.33(12) \\
\hline $\mathrm{Cl}(2)-\mathrm{Zr}(1)-\mathrm{C}(3) \# 1$ & 83.55(9) & $\mathrm{Cl}(2) \# 1-\operatorname{Zr}(1)-\mathrm{C}(3) \# 1$ & 107.92(11) \\
\hline $\mathrm{C}(1)-\mathrm{Zr}(1)-\mathrm{C}(3) \# 1$ & $54.19(13)$ & $\mathrm{C}(6)-\operatorname{Zr}(1)-\mathrm{C}(3) \# 1$ & 109.39(14) \\
\hline C(7)\#1-Zr(1)-C(3)\#1 & 115.37(13) & $\mathrm{C}(7)-\operatorname{Zr}(1)-\mathrm{C}(3) \# 1$ & $132.62(12)$ \\
\hline $\mathrm{C}(2)-\mathrm{Zr}(1)-\mathrm{C}(3) \# 1$ & $53.33(12)$ & $\mathrm{C}(2) \# 1-\operatorname{Zr}(1)-\mathrm{C}(3) \# 1$ & $32.15(12)$ \\
\hline C(3)-Zr(1)-C(3)\#1 & $31.3(2)$ & $\mathrm{Cl}(2)-\mathrm{Zr}(1)-\mathrm{C}(8)$ & $106.75(8)$ \\
\hline $\mathrm{Cl}(2) \# 1-\operatorname{Zr}(1)-\mathrm{C}(8)$ & $82.47(8)$ & $\mathrm{C}(1)-\operatorname{Zr}(1)-\mathrm{C}(8)$ & $109.88(12)$ \\
\hline C(6)-Zr(1)-C(8) & $54.66(12)$ & $\mathrm{C}(7) \# 1-\mathrm{Zr}(1)-\mathrm{C}(8)$ & $53.62(12)$ \\
\hline C(7)-Zr(1)-C(8) & $32.71(10)$ & $\mathrm{C}(2)-\mathrm{Zr}(1)-\mathrm{C}(8)$ & $115.81(11)$ \\
\hline
\end{tabular}




\begin{tabular}{|c|c|c|c|}
\hline C(2)\#1-Zr(1)-C(8) & $133.20(12)$ & $\mathrm{C}(3)-\operatorname{Zr}(1)-\mathrm{C}(8)$ & $144.60(13)$ \\
\hline C(3)\#1-Zr(1)-C(8) & $163.88(12)$ & $\mathrm{Cl}(2)-\mathrm{Zr}(1)-\mathrm{C}(8) \# 1$ & $82.47(8)$ \\
\hline Cl(2)\#1-Zr(1)-C(8)\#1 & $106.75(8)$ & $\mathrm{C}(1)-\operatorname{Zr}(1)-\mathrm{C}(8) \# 1$ & $109.88(12)$ \\
\hline C(6)-Zr(1)-C(8)\#1 & $54.66(12)$ & $\mathrm{C}(7) \# 1-\operatorname{Zr}(1)-\mathrm{C}(8) \# 1$ & $32.71(10)$ \\
\hline $\mathrm{C}(7)-\mathrm{Zr}(1)-\mathrm{C}(8) \# 1$ & $53.62(12)$ & $C(2)-\operatorname{Zr}(1)-C(8) \# 1$ & $133.20(12)$ \\
\hline $\mathrm{C}(2) \# 1-\operatorname{Zr}(1)-\mathrm{C}(8) \# 1$ & 115.81(11) & $\mathrm{C}(3)-\mathrm{Zr}(1)-\mathrm{C}(8) \# 1$ & $163.88(12)$ \\
\hline C(3)\#1-Zr(1)-C(8)\# 1 & 144.60(13) & $\mathrm{C}(8)-\mathrm{Zr}(1)-\mathrm{C}(8) \# 1$ & $31.27(15)$ \\
\hline $\mathrm{C}(2) \# 1-\mathrm{C}(1)-\mathrm{C}(2)$ & $106.5(4)$ & $\mathrm{C}(2) \# 1-\mathrm{C}(1)-\mathrm{C}(5)$ & $125.1(2)$ \\
\hline $\mathrm{C}(2)-\mathrm{C}(1)-\mathrm{C}(5)$ & $125.1(2)$ & $\mathrm{C}(2) \# 1-\mathrm{C}(1)-\mathrm{Zr}(1)$ & $74.3(2)$ \\
\hline$C(2)-C(1)-\operatorname{Zr}(1)$ & $74.3(2)$ & $\mathrm{C}(5)-\mathrm{C}(1)-\operatorname{Zr}(1)$ & 101.0(3) \\
\hline $\mathrm{C}(3)-\mathrm{C}(2)-\mathrm{C}(1)$ & 107.6(3) & $C(3)-C(2)-C(4)$ & 123.7(5) \\
\hline$C(1)-C(2)-C(4)$ & $128.5(5)$ & $\mathrm{C}(3)-\mathrm{C}(2)-\operatorname{Zr}(1)$ & 76.79(19) \\
\hline$C(1)-C(2)-\operatorname{Zr}(1)$ & $72.0(2)$ & $\mathrm{C}(4)-\mathrm{C}(2)-\mathrm{Zr}(1)$ & $121.4(3)$ \\
\hline C(3)\#1-C(3)-C(2) & 109.1(2) & C(3)\#1-C(3)-Zr(1) & 74.34(10) \\
\hline$C(2)-C(3)-\operatorname{Zr}(1)$ & 71.06(18) & $C(1)-C(5)-C(6)$ & $100.7(4)$ \\
\hline $\mathrm{C}(1)-\mathrm{C}(5)-\mathrm{C}(9)$ & $110.2(4)$ & $C(6)-C(5)-C(9)$ & 108.7(3) \\
\hline $\mathrm{C}(1)-\mathrm{C}(5)-\mathrm{C}(9) \# 1$ & $110.2(4)$ & $\mathrm{C}(6)-\mathrm{C}(5)-\mathrm{C}(9) \# 1$ & 108.7(3) \\
\hline $\mathrm{C}(9)-\mathrm{C}(5)-\mathrm{C}(9) \# 1$ & 117.1(8) & C(7)\#1-C(6)-C(7) & $106.3(4)$ \\
\hline C(7)\#1-C(6)-C(5) & $125.0(2)$ & $C(7)-C(6)-C(5)$ & $125.0(2)$ \\
\hline C(7)\#1-C(6)-Zr(1) & $73.8(2)$ & $\mathrm{C}(7)-\mathrm{C}(6)-\mathrm{Zr}(1)$ & $73.8(2)$ \\
\hline$C(5)-C(6)-\operatorname{Zr}(1)$ & $100.7(3)$ & $C(6)-C(7)-C(8)$ & 108.7(3) \\
\hline$C(6)-C(7)-\operatorname{Zr}(1)$ & $72.6(2)$ & $\mathrm{C}(8)-\mathrm{C}(7)-\operatorname{Zr}(1)$ & 77.73(18) \\
\hline C(8)\#1-C(8)-C(7) & 108.1(2) & C(8)\#1-C(8)-Zr(1) & $74.36(8)$ \\
\hline C(7)-C(8)-Zr(1) & $69.56(16)$ & & \\
\hline
\end{tabular}

Symmetry transformations used to generate equivalent atoms:

Table 3. Anisotropic displacement parameters $\left(\mathrm{A}^{\wedge} 2 \times 10^{\wedge} 3\right)$. The anisotropic displacement factor exponent takes the form: $-2 \mathrm{pi} \wedge 2\left[\mathrm{~h} \wedge 2 \mathrm{a}^{* \wedge} 2 \mathrm{U} 11+\ldots+2 \mathrm{~h} \mathrm{k} \mathrm{a}^{*} \mathrm{~b}^{*}\right.$ $\mathrm{U} 12$ ]

\begin{tabular}{lcccccc}
\hline & U11 & U22 & U33 & U23 & U13 & U12 \\
\hline $\operatorname{Zr}(1)$ & $28(1)$ & $45(1)$ & $42(1)$ & 0 & $1(1)$ & 0 \\
$\mathrm{Cl}(2)$ & $63(1)$ & $99(1)$ & $90(1)$ & $-51(1)$ & $-8(1)$ & $16(1)$ \\
$\mathrm{C}(1)$ & $31(2)$ & $69(3)$ & $50(2)$ & 0 & $-8(2)$ & 0
\end{tabular}




$\begin{array}{lrrrrrr}\mathrm{C}(2) & 41(2) & 51(2) & 84(2) & -3(2) & -21(2) & -7(1) \\ \mathrm{C}(3) & 33(1) & 117(3) & 72(2) & 21(2) & -6(2) & -17(2) \\ \mathrm{C}(4) & 109(4) & 58(3) & 251(7) & -42(4) & -98(5) & 6(3) \\ \mathrm{C}(5) & 40(3) & 160(8) & 44(3) & 0 & -1(2) & 0 \\ \mathrm{C}(6) & 30(2) & 105(4) & 43(2) & 0 & 4(2) & 0 \\ \mathrm{C}(7) & 34(1) & 77(2) & 70(2) & -25(2) & -1(1) & 12(1) \\ \mathrm{C}(8) & 29(1) & 66(2) & 61(2) & -3(2) & -5(1) & 8(1) \\ \mathrm{C}(9) & 47(4) & 153(9) & 46(4) & -30(5) & -5(3) & 7(4)\end{array}$

Table 4. Hydrogen coordinates ( x 10^4) and isotropic displacement parameters $\left(\mathrm{A}^{\wedge} 2\right.$ x 10^3).

\begin{tabular}{lcccc}
\hline & $x$ & $y$ & $z$ & $U(e q)$ \\
\hline$H(3)$ & 1793 & 6618 & 5972 & 89 \\
$\mathrm{H}(4 \mathrm{~A})$ & 3101 & 5262 & 4589 & 209 \\
$\mathrm{H}(4 \mathrm{~B})$ & 3652 & 5555 & 3064 & 209 \\
$\mathrm{H}(4 \mathrm{C})$ & 2276 & 5419 & 3216 & 209 \\
$\mathrm{H}(5 \mathrm{~A})$ & $4360(50)$ & $7860(50)$ & $1710(70)$ & $28(17)$ \\
$\mathrm{H}(7)$ & 5615 & 6046 & 3855 & 72 \\
$\mathrm{H}(8)$ & 6893 & 6609 & 5947 & 63 \\
$\mathrm{H}(9 \mathrm{~A})$ & 4955 & 6465 & 538 & 123 \\
$\mathrm{H}(9 B)$ & 3580 & 6363 & 759 & 123 \\
$\mathrm{H}(9 \mathrm{C})$ & 4456 & 5911 & 1913 & 123 \\
& & & & \\
\hline
\end{tabular}


X-Ray Report for [(Me)(H)C(1,3- $\left.\left.\mathrm{Me}_{2} \mathrm{C}_{5} \mathrm{H}_{2}\right)_{2}\right] \mathrm{ZrCl}_{2}$ (13).

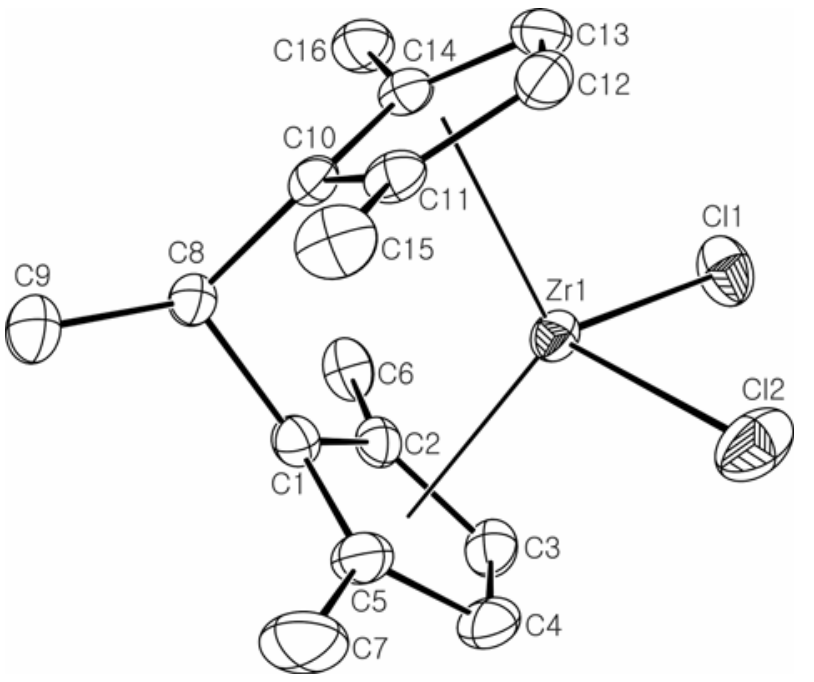

Table 1. Atomic coordinates ( $x$ 10^4) and equivalent isotropic displacement parameters $\left(\mathrm{A}^{\wedge} 2 \times 10^{\wedge} 3\right) . \mathrm{U}(\mathrm{eq})$ is defined as one third of the trace of the orthogonalized Uij tensor.

\begin{tabular}{lccrr}
\hline & $\mathrm{x}$ & $\mathrm{y}$ & $\mathrm{z}$ & $\mathrm{U}(\mathrm{eq})$ \\
\hline $\mathrm{Zr}(1)$ & $4063(1)$ & $12495(1)$ & $-2127(1)$ & $32(1)$ \\
$\mathrm{Cl}(1)$ & $3776(1)$ & $13870(1)$ & $-3129(1)$ & $55(1)$ \\
$\mathrm{Cl}(2)$ & $4156(1)$ & $9915(1)$ & $-2544(1)$ & $60(1)$ \\
$\mathrm{C}(1)$ & $3549(2)$ & $13120(5)$ & $-1062(2)$ & $35(1)$ \\
$\mathrm{C}(2)$ & $3114(2)$ & $14018(5)$ & $-1519(2)$ & $35(1)$ \\
$\mathrm{C}(3)$ & $2666(2)$ & $13018(5)$ & $-1902(2)$ & $44(1)$ \\
$\mathrm{C}(4)$ & $2802(2)$ & $11553(5)$ & $-1691(2)$ & $48(1)$ \\
$\mathrm{C}(5)$ & $3348(2)$ & $11591(5)$ & $-1164(2)$ & $42(1)$ \\
$\mathrm{C}(6)$ & $3032(2)$ & $15694(5)$ & $-1572(2)$ & $47(1)$ \\
$\mathrm{C}(7)$ & $3515(3)$ & $10172(5)$ & $-770(2)$ & $67(2)$ \\
$\mathrm{C}(8)$ & $4279(2)$ & $13727(5)$ & $-721(2)$ & $35(1)$ \\
$\mathrm{C}(9)$ & $4474(2)$ & $13139(5)$ & $-29(2)$ & $51(1)$ \\
$\mathrm{C}(10)$ & $4880(2)$ & $13467(4)$ & $-1239(2)$ & $31(1)$ \\
$\mathrm{C}(11)$ & $5222(2)$ & $12074(4)$ & $-1421(2)$ & $34(1)$ \\
$\mathrm{C}(12)$ & $5511(2)$ & $12278(5)$ & $-2053(2)$ & $40(1)$ \\
$\mathrm{C}(13)$ & $5361(2)$ & $13748(5)$ & $-2255(2)$ & $41(1)$ \\
$\mathrm{C}(14)$ & $4979(2)$ & $14504(4)$ & $-1762(2)$ & $34(1)$
\end{tabular}




\begin{tabular}{lrrrr}
$\mathrm{C}(15)$ & $5376(2)$ & $10661(5)$ & $-1031(2)$ & $51(1)$ \\
$\mathrm{C}(16)$ & $4838(3)$ & $16185(5)$ & $-1769(2)$ & $49(1)$ \\
$\mathrm{Zr}(2)$ & $2143(1)$ & $6837(1)$ & $874(1)$ & $36(1)$ \\
$\mathrm{Cl}(3)$ & $3248(1)$ & $6730(1)$ & $178(1)$ & $57(1)$ \\
$\mathrm{Cl}(4)$ & $2322(1)$ & $9261(1)$ & $1411(1)$ & $73(1)$ \\
$\mathrm{C}(17)$ & $1612(2)$ & $4695(5)$ & $1454(2)$ & $36(1)$ \\
$\mathrm{C}(18)$ & $1804(2)$ & $5749(5)$ & $1949(2)$ & $43(1)$ \\
$\mathrm{C}(19)$ & $2609(3)$ & $5860(6)$ & $1977(2)$ & $50(1)$ \\
$\mathrm{C}(21)$ & $2307(3)$ & $4155(5)$ & $1188(2)$ & $46(1)$ \\
$\mathrm{C}(20)$ & $2907(3)$ & $4915(5)$ & $1523(2)$ & $52(1)$ \\
$\mathrm{C}(22)$ & $1313(3)$ & $6533(5)$ & $2430(2)$ & $59(1)$ \\
$\mathrm{C}(23)$ & $2465(3)$ & $2918(5)$ & $710(2)$ & $65(1)$ \\
$\mathrm{C}(24)$ & $815(2)$ & $4617(5)$ & $1130(2)$ & $40(1)$ \\
$\mathrm{C}(25)$ & $519(3)$ & $3071(5)$ & $918(2)$ & $60(1)$ \\
$\mathrm{C}(26)$ & $868(2)$ & $5848(5)$ & $601(2)$ & $41(1)$ \\
$\mathrm{C}(27)$ & $1275(2)$ & $5778(5)$ & $5(2)$ & $44(1)$ \\
$\mathrm{C}(28)$ & $1412(3)$ & $7267(6)$ & $-198(2)$ & $55(1)$ \\
$\mathrm{C}(29)$ & $1102(3)$ & $8255(6)$ & $243(2)$ & $58(1)$ \\
$\mathrm{C}(30)$ & $748(3)$ & $7415(5)$ & $739(2)$ & $48(1)$ \\
$\mathrm{C}(31)$ & $1427(3)$ & $4446(6)$ & $-442(2)$ & $63(1)$ \\
$\mathrm{C}(32)$ & $260(3)$ & $8126(6)$ & $1253(2)$ & $75(2)$ \\
& & & & \\
\hline
\end{tabular}

Table 2. Bond lengths [A] and angles [deg] .

\begin{tabular}{llll}
\hline $\operatorname{Zr}(1)-C l(1)$ & $2.4338(11)$ & $\operatorname{Zr}(1)-C l(2)$ & $2.4469(12)$ \\
$\operatorname{Zr}(1)-C(1)$ & $2.448(4)$ & $\operatorname{Zr}(1)-C(10)$ & $2.448(3)$ \\
$\operatorname{Zr}(1)-C(11)$ & $2.498(3)$ & $\operatorname{Zr}(1)-C(5)$ & $02.498(4)$ \\
$\operatorname{Zr}(1)-C(2)$ & $2.502(4)$ & $\operatorname{Zr}(1)-C(14)$ & $2.502(4)$ \\
$\operatorname{Zr}(1)-C(4)$ & $2.551(4)$ & $\operatorname{Zr}(1)-C(3)$ & $2.551(4)$ \\
$\operatorname{Zr}(1)-C(12)$ & $2.552(4)$ & $\operatorname{Zr}(1)-C(13)$ & $2.556(4)$ \\
$\mathrm{C}(1)-C(5)$ & $1.414(5)$ & $\mathrm{C}(1)-C(2)$ & $1.436(5)$ \\
$\mathrm{C}(1)-C(8)$ & $1.544(5)$ & $\mathrm{C}(2)-C(3)$ & $1.411(5)$ \\
$\mathrm{C}(2)-C(6)$ & $1.496(5)$ & $\mathrm{C}(3)-C(4)$ & $1.387(6)$ \\
$\mathrm{C}(4)-C(5)$ & $1.428(5)$ & $\mathrm{C}(5)-C(7)$ & $1.519(6)$ \\
$\mathrm{C}(8)-C(10)$ & $1.533(5)$ & $\mathrm{C}(8)-C(9)$ & $1.543(5)$
\end{tabular}




\begin{tabular}{|c|c|c|c|}
\hline $\mathrm{C}(10)-\mathrm{C}(11)$ & $1.426(5)$ & $\mathrm{C}(10)-\mathrm{C}(14)$ & $1.428(5)$ \\
\hline $\mathrm{C}(11)-\mathrm{C}(12)$ & $1.415(5)$ & C(11)-C(15) & $1.506(5)$ \\
\hline C(12)-C(13) & $1.390(6)$ & C(13)-C(14) & $1.398(5)$ \\
\hline$C(14)-C(16)$ & $1.510(5)$ & $\operatorname{Zr}(2)-\mathrm{Cl}(4)$ & $2.4304(13)$ \\
\hline $\operatorname{Zr}(2)-C(17)$ & $2.437(4)$ & $\operatorname{Zr}(2)-\mathrm{Cl}(3)$ & $2.4409(12)$ \\
\hline $\operatorname{Zr}(2)-C(26)$ & $2.457(4)$ & $\operatorname{Zr}(2)-C(21)$ & $2.476(4)$ \\
\hline $\operatorname{Zr}(2)-C(18)$ & $2.492(4)$ & $\operatorname{Zr}(2)-C(27)$ & $2.501(4)$ \\
\hline $\operatorname{Zr}(2)-C(30)$ & $2.512(4)$ & $\operatorname{Zr}(2)-C(20)$ & $2.527(4)$ \\
\hline Zr(2)-C(19) & $2.540(4)$ & $\operatorname{Zr}(2)-C(29)$ & $2.547(4)$ \\
\hline $\operatorname{Zr}(2)-C(28)$ & $2.549(4)$ & C(17)-C(18) & $1.415(5)$ \\
\hline $\mathrm{C}(17)-\mathrm{C}(21)$ & $1.432(6)$ & C(17)-C(24) & $1.537(5)$ \\
\hline C(18)-C(19 & $1.417(6)$ & C(18)-C(22) & $1.499(6)$ \\
\hline C(19)-C(20) & $1.366(6)$ & $C(21)-C(20)$ & $1.414(6)$ \\
\hline C(21)-C(23) & $1.502(6)$ & C(24)-C(25) & $1.524(5)$ \\
\hline$C(24)-C(26)$ & $1.543(5)$ & $\mathrm{C}(26)-\mathrm{C}(27)$ & $1.432(6)$ \\
\hline C(26)-C(30) & $1.433(6)$ & $\mathrm{C}(27)-\mathrm{C}(28)$ & $1.406(6)$ \\
\hline $\mathrm{C}(27)-\mathrm{C}(31)$ & $1.522(6)$ & C(28)-C(29) & $1.382(6)$ \\
\hline C(29)-C(30) & $1.416(6)$ & C(30)-C(32) & $1.513(6)$ \\
\hline Cl(1)-Zr(1)-Cl(2) & $100.69(4)$ & $\mathrm{Cl}(1)-\mathrm{Zr}(1)-\mathrm{C}(1)$ & 124.61(10) \\
\hline $\mathrm{Cl}(2)-\operatorname{Zr}(1)-\mathrm{C}(1)$ & 123.57(10) & $\mathrm{Cl}(1)-\mathrm{Zr}(1)-\mathrm{C}(10)$ & 123.98(9) \\
\hline $\mathrm{Cl}(2)-\mathrm{Zr}(1)-\mathrm{C}(10)$ & 123.11(9) & $C(1)-\operatorname{Zr}(1)-C(10)$ & $58.58(13)$ \\
\hline Cl(1)-Zr(1)-C(11) & $135.57(10)$ & $\mathrm{Cl}(2)-\operatorname{Zr}(1)-\mathrm{C}(11)$ & $90.19(10)$ \\
\hline $\mathrm{C}(1)-\operatorname{Zr}(1)-\mathrm{C}(11)$ & 80.21(13) & $C(10)-\operatorname{Zr}(1)-C(11)$ & $33.50(12)$ \\
\hline $\mathrm{Cl}(1)-\mathrm{Zr}(1)-\mathrm{C}(5)$ & 136.98(10) & $\mathrm{Cl}(2)-\mathrm{Zr}(1)-\mathrm{C}(5)$ & $90.97(10)$ \\
\hline C(1)-Zr(1)-C(5) & 33.19(13) & $\mathrm{C}(10)-\operatorname{Zr}(1)-\mathrm{C}(5)$ & $79.70(13)$ \\
\hline $\mathrm{C}(11)-\operatorname{Zr}(1)-\mathrm{C}(5)$ & 84.98(13) & $\mathrm{Cl}(1)-\mathrm{Zr}(1)-\mathrm{C}(2)$ & 91.41(9) \\
\hline $\mathrm{Cl}(2)-\mathrm{Zr}(1)-\mathrm{C}(2)$ & $136.80(10)$ & $\mathrm{C}(1)-\operatorname{Zr}(1)-\mathrm{C}(2)$ & 33.69(11) \\
\hline $\mathrm{C}(11)-\operatorname{Zr}(1)-\mathrm{C}(2)$ & 109.61(12) & $\mathrm{C}(5)-\operatorname{Zr}(1)-\mathrm{C}(2)$ & $54.85(13)$ \\
\hline $\mathrm{Cl}(1)-\operatorname{Zr}(1)-\mathrm{C}(14)$ & $90.87(9)$ & Cl(2)-Zr(1)-C(14) & $136.12(10)$ \\
\hline $\mathrm{C}(1)-\mathrm{Zr}(1)-\mathrm{C}(14)$ & 79.70(13) & $\mathrm{C}(10)-\operatorname{Zr}(1)-\mathrm{C}(14)$ & $33.50(12)$ \\
\hline $\mathrm{C}(11)-\mathrm{Zr}(1)-\mathrm{C}(14)$ & $54.77(12)$ & $C(5)-\operatorname{Zr}(1)-C(14)$ & 108.90(13) \\
\hline $\mathrm{C}(2)-\operatorname{Zr}(1)-\mathrm{C}(14)$ & 84.24(13) & $\mathrm{Cl}(1)-\operatorname{Zr}(1)-\mathrm{C}(4)$ & $107.26(10)$ \\
\hline $\mathrm{Cl}(2)-\mathrm{Zr}(1)-\mathrm{C}(4)$ & 83.31(11) & $\mathrm{C}(1)-\operatorname{Zr}(1)-\mathrm{C}(4)$ & $54.40(13)$ \\
\hline $\mathrm{C}(10)-\operatorname{Zr}(1)-\mathrm{C}(4)$ & 110.63(13) & $C(11)-\operatorname{Zr}(1)-C(4)$ & $116.80(13)$ \\
\hline C(5)-Zr(1)-C(4) & $32.83(12)$ & $\mathrm{C}(2)-\mathrm{Zr}(1)-\mathrm{C}(4)$ & $53.54(14)$ \\
\hline
\end{tabular}




\begin{tabular}{|c|c|c|c|}
\hline$C(14)-\operatorname{Zr}(1)-C(4)$ & 133.32(14) & $\mathrm{Cl}(1)-\operatorname{Zr}(1)-\mathrm{C}(3)$ & 83.19(10) \\
\hline Cl(2)-Zr(1)-C(3) & $107.79(10)$ & C(1)-Zr(1)-C(3) & $54.54(12)$ \\
\hline $\mathrm{C}(10)-\operatorname{Zr}(1)-\mathrm{C}(3)$ & $110.54(13)$ & $\mathrm{C}(11)-\operatorname{Zr}(1)-\mathrm{C}(3)$ & $134.17(13)$ \\
\hline C(5)-Zr(1)-C(3) & 53.89(13) & $\mathrm{C}(2)-\mathrm{Zr}(1)-\mathrm{C}(3)$ & $32.41(12)$ \\
\hline C(14)-Zr(1)-C(3) & $115.59(14)$ & $\mathrm{C}(4)-\mathrm{Zr}(1)-\mathrm{C}(3)$ & 31.55(13) \\
\hline Cl(1)-Zr(1)-C(12) & $105.87(10)$ & $\mathrm{Cl}(2)-\mathrm{Zr}(1)-\mathrm{C}(12)$ & $82.87(10)$ \\
\hline $\mathrm{C}(1)-\operatorname{Zr}(1)-\mathrm{C}(12)$ & $110.68(12)$ & $\mathrm{C}(10)-\mathrm{Zr}(1)-\mathrm{C}(12)$ & $54.32(13)$ \\
\hline $\mathrm{C}(11)-\mathrm{Zr}(1)-\mathrm{C}(12)$ & $32.53(12)$ & C(5)-Zr(1)-C(12) & $116.67(13)$ \\
\hline C(2)-Zr(1)-C(12) & 133.37(12) & $\mathrm{C}(14)-\mathrm{Zr}(1)-\mathrm{C}(12)$ & 53.29(13) \\
\hline $\mathrm{C}(4)-\operatorname{Zr}(1)-\mathrm{C}(12)$ & $145.90(14)$ & $\mathrm{C}(3)-\operatorname{Zr}(1)-\mathrm{C}(12)$ & 164.81(12) \\
\hline Cl(1)-Zr(1)-C(13) & 82.09(9) & $\mathrm{Cl}(2)-\mathrm{Zr}(1)-\mathrm{C}(13)$ & 107.59(11) \\
\hline C(1)-Zr(1)-C(13) & 110.09(13) & $\mathrm{C}(10)-\operatorname{Zr}(1)-\mathrm{C}(13)$ & $54.24(13)$ \\
\hline C(11)-Zr(1)-C(13) & $53.67(13)$ & C(5)-Zr(1)-C(13) & $133.46(12)$ \\
\hline C(2)-Zr(1)-C(13) & $115.13(14)$ & $\mathrm{C}(14)-\mathrm{Zr}(1)-\mathrm{C}(13)$ & $32.06(12)$ \\
\hline $\mathrm{C}(4)-\mathrm{Zr}(1)-\mathrm{C}(13)$ & $164.44(13)$ & C(3)-Zr(1)-C(13) & $143.58(15)$ \\
\hline C(12)-Zr(1)-C(13) & $31.57(12)$ & $C(5)-C(1)-C(2)$ & 107.9(3) \\
\hline C(5)-C(1)-C(8) & $127.0(3)$ & $C(2)-C(1)-C(8)$ & 121.8(3) \\
\hline C(5)-C(1)-Zr(1) & $75.4(2)$ & $\mathrm{C}(2)-\mathrm{C}(1)-\mathrm{Zr}(1)$ & $75.2(2)$ \\
\hline $\mathrm{C}(8)-\mathrm{C}(1)-\operatorname{Zr}(1)$ & 99.3(2) & C(3)-C(2)-C(1) & 107.3(4) \\
\hline $\mathrm{C}(3)-\mathrm{C}(2)-\mathrm{C}(6)$ & 122.1(3) & $C(1)-C(2)-C(6)$ & 130.2(3) \\
\hline C(3)-C(2)-Zr(1) & $75.7(2)$ & $\mathrm{C}(1)-\mathrm{C}(2)-\mathrm{Zr}(1)$ & $71.1(2)$ \\
\hline$C(6)-C(2)-Z r(1)$ & 124.3(3) & $C(4)-C(3)-C(2)$ & $108.9(4)$ \\
\hline $\mathrm{C}(4)-\mathrm{C}(3)-\operatorname{Zr}(1)$ & $74.2(2)$ & $\mathrm{C}(2)-\mathrm{C}(3)-\operatorname{Zr}(1)$ & $71.9(2)$ \\
\hline $\mathrm{C}(3)-\mathrm{C}(4)-\mathrm{C}(5)$ & $108.8(4)$ & $\mathrm{C}(3)-\mathrm{C}(4)-\mathrm{Zr}(1)$ & $74.2(3)$ \\
\hline$C(5)-C(4)-\operatorname{Zr}(1)$ & $71.6(2)$ & $C(1)-C(5)-C(4)$ & $107.2(4)$ \\
\hline $\mathrm{C}(1)-\mathrm{C}(5)-\mathrm{C}(7)$ & $132.0(4)$ & $C(4)-C(5)-C(7)$ & $120.1(4)$ \\
\hline $\mathrm{C}(1)-\mathrm{C}(5)-\operatorname{Zr}(1)$ & $71.5(2)$ & $C(4)-C(5)-\operatorname{Zr}(1)$ & $75.6(2)$ \\
\hline$C(7)-C(5)-\operatorname{Zr}(1)$ & $126.2(3)$ & $\mathrm{C}(10)-\mathrm{C}(8)-\mathrm{C}(9)$ & 116.6(3) \\
\hline$C(10)-C(8)-C(1)$ & 102.2(3) & $\mathrm{C}(9)-\mathrm{C}(8)-\mathrm{C}(1)$ & 117.6(3) \\
\hline$C(11)-C(10)-C(14)$ & $107.4(3)$ & $\mathrm{C}(11)-\mathrm{C}(10)-\mathrm{C}(8)$ & $127.8(4)$ \\
\hline $\mathrm{C}(14)-\mathrm{C}(10)-\mathrm{C}(8)$ & 121.6(3) & $C(11)-C(10)-\operatorname{Zr}(1)$ & $75.1(2)$ \\
\hline C(14)-C(10)-Zr(1) & $75.3(2)$ & $\mathrm{C}(8)-\mathrm{C}(10)-\mathrm{Zr}(1)$ & $99.7(2)$ \\
\hline$C(12)-C(11)-C(10)$ & $107.0(3)$ & $C(12)-C(11)-C(15)$ & $121.9(4)$ \\
\hline$C(10)-C(11)-C(15)$ & $130.6(4)$ & $\mathrm{C}(12)-\mathrm{C}(11)-\operatorname{Zr}(1)$ & $75.9(2)$ \\
\hline C(10)-C(11)-Zr(1) & 71.35(19) & $C(15)-C(11)-\operatorname{Zr}(1)$ & $124.4(3)$ \\
\hline$C(13)-C(12)-C(11)$ & $108.9(4)$ & $\mathrm{C}(13)-\mathrm{C}(12)-\operatorname{Zr}(1)$ & $74.4(2)$ \\
\hline
\end{tabular}




\begin{tabular}{|c|c|c|c|}
\hline $\mathrm{C}(11)-\mathrm{C}(12)-\operatorname{Zr}(1)$ & $71.6(2)$ & $C(12)-C(13)-C(14)$ & $108.9(4)$ \\
\hline $\mathrm{C}(12)-\mathrm{C}(13)-\operatorname{Zr}(1)$ & $74.1(2)$ & $C(14)-C(13)-\operatorname{Zr}(1)$ & $71.8(2)$ \\
\hline $\mathrm{C}(13)-\mathrm{C}(14)-\mathrm{C}(10)$ & $107.8(4)$ & $C(13)-C(14)-C(16)$ & 123.2(4) \\
\hline$C(10)-C(14)-C(16)$ & $128.2(4)$ & $C(13)-C(14)-\operatorname{Zr}(1)$ & $76.1(2)$ \\
\hline C(10)-C(14)-Zr(1) & $71.2(2)$ & $\mathrm{C}(16)-\mathrm{C}(14)-\operatorname{Zr}(1)$ & $126.4(3)$ \\
\hline $\mathrm{Cl}(4)-\mathrm{Zr}(2)-\mathrm{C}(17)$ & $120.90(10)$ & $\mathrm{Cl}(4)-\mathrm{Zr}(2)-\mathrm{Cl}(3)$ & $101.73(5)$ \\
\hline $\mathrm{C}(17)-\mathrm{Zr}(2)-\mathrm{Cl}(3)$ & $125.13(11)$ & $\mathrm{Cl}(4)-\mathrm{Zr}(2)-\mathrm{C}(26)$ & $121.71(11)$ \\
\hline $\mathrm{C}(17)-\operatorname{Zr}(2)-\mathrm{C}(26)$ & 58.39(13) & $\mathrm{Cl}(3)-\operatorname{Zr}(2)-\mathrm{C}(26)$ & $125.90(10)$ \\
\hline $\mathrm{Cl}(4)-\mathrm{Zr}(2)-\mathrm{C}(21)$ & 135.77(11) & $\mathrm{C}(17)-\operatorname{Zr}(2)-\mathrm{C}(21)$ & 33.88(13) \\
\hline $\mathrm{Cl}(3)-\operatorname{Zr}(2)-\mathrm{C}(21)$ & $91.51(11)$ & $C(26)-\operatorname{Zr}(2)-C(21)$ & $79.46(14)$ \\
\hline $\mathrm{Cl}(4)-\mathrm{Zr}(2)-\mathrm{C}(18)$ & 88.37(11) & $C(17)-\operatorname{Zr}(2)-C(18)$ & 33.35(13) \\
\hline Cl(3)-Zr(2)-C(18) & $135.56(11)$ & $C(26)-\operatorname{Zr}(2)-C(18)$ & $80.10(13)$ \\
\hline $\mathrm{C}(21)-\mathrm{Zr}(2)-\mathrm{C}(18)$ & $55.08(15)$ & $\mathrm{Cl}(4)-\mathrm{Zr}(2)-\mathrm{C}(27)$ & $136.44(11)$ \\
\hline $\mathrm{C}(17)-\mathrm{Zr}(2)-\mathrm{C}(27)$ & 79.73(13) & $\mathrm{Cl}(3)-\operatorname{Zr}(2)-\mathrm{C}(27)$ & $92.76(11)$ \\
\hline $\mathrm{C}(26)-\mathrm{Zr}(2)-\mathrm{C}(27)$ & 33.56(13) & $\mathrm{C}(21)-\operatorname{Zr}(2)-\mathrm{C}(27)$ & 83.74(14) \\
\hline $\mathrm{C}(18)-\mathrm{Zr}(2)-\mathrm{C}(27)$ & 109.35(14) & $\mathrm{Cl}(4)-\operatorname{Zr}(2)-\mathrm{C}(30)$ & 89.16(11) \\
\hline C(17)-Zr(2)-C(30) & $80.10(14)$ & Cl(3)-Zr(2)-C(30) & $136.98(11)$ \\
\hline $\mathrm{C}(26)-\mathrm{Zr}(2)-\mathrm{C}(30)$ & $33.49(14)$ & $\mathrm{C}(21)-\operatorname{Zr}(2)-\mathrm{C}(30)$ & 109.33(15) \\
\hline C(18)-Zr(2)-C(30) & $85.57(14)$ & $\mathrm{C}(27)-\operatorname{Zr}(2)-\mathrm{C}(30)$ & $54.64(14)$ \\
\hline Cl(4)-Zr(2)-C(20) & $107.15(12)$ & $C(17)-\operatorname{Zr}(2)-C(20)$ & $54.78(14)$ \\
\hline Cl(3)-Zr(2)-C(20) & $82.12(12)$ & $C(26)-\operatorname{Zr}(2)-C(20)$ & $110.47(14)$ \\
\hline $\mathrm{C}(21)-\operatorname{Zr}(2)-\mathrm{C}(20)$ & 32.83(13) & $C(18)-\operatorname{Zr}(2)-C(20)$ & 53.73(15) \\
\hline$C(27)-\operatorname{Zr}(2)-C(20)$ & $115.48(15)$ & $C(30)-\operatorname{Zr}(2)-C(20)$ & 134.38(15) \\
\hline $\mathrm{Cl}(4)-\mathrm{Zr}(2)-\mathrm{C}(19)$ & 82.04(12) & $\mathrm{C}(17)-\operatorname{Zr}(2)-\mathrm{C}(19)$ & $54.49(13)$ \\
\hline Cl(3)-Zr(2)-C(19) & 105.37(11) & $C(26)-\operatorname{Zr}(2)-C(19)$ & $110.75(14)$ \\
\hline $\mathrm{C}(21)-\operatorname{Zr}(2)-\mathrm{C}(19)$ & 53.74(15) & $\mathrm{C}(18)-\operatorname{Zr}(2)-\mathrm{C}(19)$ & $32.70(13)$ \\
\hline$C(27)-\operatorname{Zr}(2)-C(19)$ & 133.28(15) & $C(30)-\operatorname{Zr}(2)-C(19)$ & $117.38(15)$ \\
\hline C(20)-Zr(2)-C(19) & $31.29(14)$ & $\mathrm{Cl}(4)-\mathrm{Zr}(2)-\mathrm{C}(29)$ & 82.99(12) \\
\hline C(17)-Zr(2)-C(29) & 110.67(15) & Cl(3)-Zr(2)-C(29) & $107.06(12)$ \\
\hline C(26)-Zr(2)-C(29) & $54.56(14)$ & $C(21)-\operatorname{Zr}(2)-C(29)$ & 133.09(15) \\
\hline C(18)-Zr(2)-C(29) & 117.15(16) & $\mathrm{C}(27)-\operatorname{Zr}(2)-\mathrm{C}(29)$ & $53.45(15)$ \\
\hline C(30)-Zr(2)-C(29) & $32.51(14)$ & $C(20)-\operatorname{Zr}(2)-C(29)$ & $165.00(15)$ \\
\hline C(19)-Zr(2)-C(29) & 146.39(17) & $\mathrm{Cl}(4)-\operatorname{Zr}(2)-\mathrm{C}(28)$ & $108.43(12)$ \\
\hline $\mathrm{C}(17)-\operatorname{Zr}(2)-\mathrm{C}(28)$ & $110.28(14)$ & $\mathrm{Cl}(3)-\operatorname{Zr}(2)-\mathrm{C}(28)$ & 83.77(12) \\
\hline$C(26)-\operatorname{Zr}(2)-C(28)$ & $54.48(14)$ & $C(21)-\operatorname{Zr}(2)-C(28)$ & 114.91(15) \\
\hline $\mathrm{C}(18)-\operatorname{Zr}(2)-\mathrm{C}(28)$ & $134.07(15)$ & $\mathrm{C}(27)-\operatorname{Zr}(2)-\mathrm{C}(28)$ & 32.32(13) \\
\hline
\end{tabular}




\begin{tabular}{|c|c|c|c|}
\hline$C(30)-\operatorname{Zr}(2)-C(28)$ & $53.48(15)$ & $C(20)-\operatorname{Zr}(2)-C(28)$ & 143.67(17) \\
\hline C(19)-Zr(2)-C(28) & 164.75(15) & $\mathrm{C}(29)-\mathrm{Zr}(2)-\mathrm{C}(28)$ & $31.46(14)$ \\
\hline $\mathrm{C}(18)-\mathrm{C}(17)-\mathrm{C}(21)$ & $107.6(4)$ & $\mathrm{C}(18)-\mathrm{C}(17)-\mathrm{C}(24)$ & $122.6(4)$ \\
\hline $\mathrm{C}(21)-\mathrm{C}(17)-\mathrm{C}(24)$ & $126.7(4)$ & $\mathrm{C}(18)-\mathrm{C}(17)-\mathrm{Zr}(2)$ & $75.5(2)$ \\
\hline $\mathrm{C}(21)-\mathrm{C}(17)-\operatorname{Zr}(2)$ & $74.5(2)$ & $C(24)-C(17)-\operatorname{Zr}(2)$ & $100.4(2)$ \\
\hline C(17)-C(18)-C(19) & 107.3(4) & $\mathrm{C}(17)-\mathrm{C}(18)-\mathrm{C}(22)$ & $130.4(4)$ \\
\hline$C(19)-C(18)-C(22)$ & 122.1(4) & $\mathrm{C}(17)-\mathrm{C}(18)-\operatorname{Zr}(2)$ & $71.2(2)$ \\
\hline C(19)-C(18)-Zr(2) & $75.5(2)$ & $\mathrm{C}(22)-\mathrm{C}(18)-\mathrm{Zr}(2)$ & $124.0(3)$ \\
\hline $\mathrm{C}(20)-\mathrm{C}(19)-\mathrm{C}(18)$ & 109.1(4) & $C(20)-C(19)-\operatorname{Zr}(2)$ & $73.8(2)$ \\
\hline C(18)-C(19)-Zr(2) & $71.8(2)$ & $C(20)-C(21)-C(17)$ & $106.8(4)$ \\
\hline$C(20)-C(21)-C(23)$ & 121.1(4) & $C(17)-C(21)-C(23)$ & $131.7(4)$ \\
\hline C(20)-C(21)-Zr(2) & $75.6(2)$ & $\mathrm{C}(17)-\mathrm{C}(21)-\operatorname{Zr}(2)$ & $71.6(2)$ \\
\hline $\mathrm{C}(23)-\mathrm{C}(21)-\operatorname{Zr}(2)$ & 123.5(3) & $C(19)-C(20)-C(21)$ & 109.3(4) \\
\hline$C(19)-C(20)-\operatorname{Zr}(2)$ & $74.9(3)$ & $C(21)-C(20)-\operatorname{Zr}(2)$ & $71.6(2)$ \\
\hline $\mathrm{C}(25)-\mathrm{C}(24)-\mathrm{C}(17)$ & $117.7(4)$ & $C(25)-C(24)-C(26)$ & 117.4(3) \\
\hline$C(17)-C(24)-C(26)$ & 101.7(3) & $C(27)-C(26)-C(30)$ & $106.9(4)$ \\
\hline $\mathrm{C}(27)-\mathrm{C}(26)-\mathrm{C}(24)$ & $127.6(4)$ & $C(30)-C(26)-C(24)$ & $122.2(4)$ \\
\hline C(27)-C(26)-Zr(2) & $74.9(2)$ & $\mathrm{C}(30)-\mathrm{C}(26)-\operatorname{Zr}(2)$ & $75.4(2)$ \\
\hline $\mathrm{C}(24)-\mathrm{C}(26)-\operatorname{Zr}(2)$ & $99.3(2)$ & $\mathrm{C}(28)-\mathrm{C}(27)-\mathrm{C}(26)$ & $107.8(4)$ \\
\hline$C(28)-C(27)-C(31)$ & $120.9(4)$ & $C(26)-C(27)-C(31)$ & $130.3(4)$ \\
\hline $\mathrm{C}(28)-\mathrm{C}(27)-\operatorname{Zr}(2)$ & $75.7(2)$ & $C(26)-C(27)-\operatorname{Zr}(2)$ & $71.6(2)$ \\
\hline $\mathrm{C}(31)-\mathrm{C}(27)-\operatorname{Zr}(2)$ & 127.4(3) & $C(29)-C(28)-C(27)$ & $109.0(4)$ \\
\hline C(29)-C(28)-Zr(2) & $74.2(2)$ & $\mathrm{C}(27)-\mathrm{C}(28)-\operatorname{Zr}(2)$ & $71.9(2)$ \\
\hline C(28)-C(29)-C(30) & 109.0(4) & $\mathrm{C}(28)-\mathrm{C}(29)-\operatorname{Zr}(2)$ & 74.4(3) \\
\hline C(30)-C(29)-Zr(2) & $72.4(2)$ & $C(29)-C(30)-C(26)$ & 107.3(4) \\
\hline $\mathrm{C}(29)-\mathrm{C}(30)-\mathrm{C}(32)$ & 123.2(5) & $C(26)-C(30)-C(32)$ & $129.0(4)$ \\
\hline C(29)-C(30)-Zr(2) & 75.1(3) & $\mathrm{C}(26)-\mathrm{C}(30)-\mathrm{Zr}(2)$ & $71.1(2)$ \\
\hline C(32)-C(30)-Zr(2) & $125.2(3)$ & & \\
\hline
\end{tabular}

Symmetry transformations used to generate equivalent atoms:

Table 3. Anisotropic displacement parameters $\left(\mathrm{A}^{\wedge} 2 \times 10^{\wedge} 3\right)$. The anisotropic displacement factor exponent takes the form:

$-2 \mathrm{pi}^{\wedge} 2\left[\mathrm{~h} \wedge 2 \mathrm{a}^{* \wedge} \mathrm{U} 11+\ldots+2 \mathrm{~h} \mathrm{k} \mathrm{a*} \mathrm{b}^{*} \mathrm{U} 12\right]$

\begin{tabular}{llllll}
\hline U11 & U22 & U33 & U23 & U13 & U12
\end{tabular}




\begin{tabular}{|c|c|c|c|c|c|c|}
\hline $\operatorname{Zr}(1)$ & $30(1)$ & $34(1)$ & $33(1)$ & $-3(1)$ & $-4(1)$ & $0(1)$ \\
\hline $\mathrm{Cl}(1)$ & $55(1)$ & $75(1)$ & $36(1)$ & $7(1)$ & $-3(1)$ & $16(1)$ \\
\hline $\mathrm{Cl}(2)$ & $49(1)$ & $45(1)$ & $86(1)$ & $-23(1)$ & $-4(1)$ & $1(1)$ \\
\hline $\mathrm{C}(1)$ & $36(2)$ & $38(2)$ & $29(2)$ & $3(2)$ & $1(2)$ & $-1(2)$ \\
\hline C(2) & $29(2)$ & $45(3)$ & $30(2)$ & $-1(2)$ & $1(2)$ & $4(2)$ \\
\hline C(3) & $30(2)$ & $60(3)$ & $41(2)$ & $-6(2)$ & $-5(2)$ & $5(2)$ \\
\hline C(4) & $30(3)$ & $57(3)$ & $58(3)$ & $-11(2)$ & $2(2)$ & $-10(2)$ \\
\hline C(5) & $40(3)$ & $40(3)$ & $47(3)$ & 2(2) & $0(2)$ & $-9(2)$ \\
\hline C(6) & $54(3)$ & $49(3)$ & $39(2)$ & $4(2)$ & $1(2)$ & $15(2)$ \\
\hline C(7) & $74(4)$ & $51(3)$ & $76(3)$ & $24(3)$ & $-2(3)$ & $-22(3)$ \\
\hline C(8) & $37(2)$ & $38(2)$ & $29(2)$ & $1(2)$ & $-3(2)$ & $-4(2)$ \\
\hline C(9) & 56(3) & $60(3)$ & $35(2)$ & $3(2)$ & $-9(2)$ & $-4(3)$ \\
\hline$C(10)$ & $30(2)$ & $32(3)$ & $31(2)$ & $0(2)$ & $-6(2)$ & $-3(2)$ \\
\hline $\mathrm{C}(11)$ & $27(2)$ & $34(3)$ & $41(2)$ & $4(2)$ & $-8(2)$ & $-1(2)$ \\
\hline $\mathrm{C}(12)$ & $32(2)$ & $45(3)$ & $44(3)$ & $-12(2)$ & $0(2)$ & $1(2)$ \\
\hline C(13) & $33(2)$ & $48(3)$ & $41(2)$ & $8(2)$ & $2(2)$ & $-8(2)$ \\
\hline$C(14)$ & $32(2)$ & $31(2)$ & $39(2)$ & $3(2)$ & $-4(2)$ & $-6(2)$ \\
\hline$C(15)$ & $47(3)$ & $45(3)$ & $62(3)$ & $7(2)$ & $-7(2)$ & $4(2)$ \\
\hline$C(16)$ & $50(3)$ & $42(3)$ & $55(3)$ & $5(2)$ & 2(2) & $-9(2)$ \\
\hline $\operatorname{Zr}(2)$ & $40(1)$ & $37(1)$ & $33(1)$ & $4(1)$ & $-2(1)$ & $-8(1)$ \\
\hline $\mathrm{Cl}(3)$ & $53(1)$ & $71(1)$ & $46(1)$ & $4(1)$ & $11(1)$ & $-11(1)$ \\
\hline $\mathrm{Cl}(4)$ & $106(1)$ & $47(1)$ & $66(1)$ & $-10(1)$ & $-4(1)$ & $-21(1)$ \\
\hline$C(17)$ & $37(3)$ & $36(3)$ & $35(2)$ & $5(2)$ & $4(2)$ & $-7(2)$ \\
\hline $\mathrm{C}(18)$ & $44(3)$ & $49(3)$ & $35(2)$ & $10(2)$ & $0(2)$ & $-9(2)$ \\
\hline C(19) & $45(3)$ & $68(4)$ & $38(3)$ & $16(2)$ & $-7(2)$ & $-17(3)$ \\
\hline $\mathrm{C}(21)$ & $50(3)$ & $36(3)$ & $52(3)$ & $16(2)$ & $1(2)$ & $1(2)$ \\
\hline$C(20)$ & $36(3)$ & $68(4)$ & $52(3)$ & 23(3) & $-5(2)$ & $-4(3)$ \\
\hline $\mathrm{C}(22)$ & $63(3)$ & $72(4)$ & 41(3) & $-6(2)$ & $7(2)$ & $-17(3)$ \\
\hline$C(23)$ & $64(4)$ & $50(3)$ & $83(4)$ & $6(3)$ & $12(3)$ & $9(3)$ \\
\hline$C(24)$ & $38(3)$ & $42(3)$ & $40(2)$ & $-2(2)$ & $6(2)$ & $-10(2)$ \\
\hline$C(25)$ & $59(3)$ & $59(3)$ & 61(3) & $-1(3)$ & $3(2)$ & $-24(3)$ \\
\hline$C(26)$ & 33(3) & $50(3)$ & $38(2)$ & $-1(2)$ & $-7(2)$ & $-4(2)$ \\
\hline $\mathrm{C}(27)$ & 39(3) & 51(3) & 41(3) & $1(2)$ & $-7(2)$ & $-4(2)$ \\
\hline$C(28)$ & $58(3)$ & $66(4)$ & $40(3)$ & $13(2)$ & $-8(2)$ & $-12(3)$ \\
\hline C(29) & $65(4)$ & $43(3)$ & $65(3)$ & 19(3) & $-18(3)$ & $-1(3)$ \\
\hline
\end{tabular}




$\begin{array}{rrrrrrr}\mathrm{C}(30) & 46(3) & 45(3) & 51(3) & 4(2) & -10(2) & 0(2) \\ \mathrm{C}(31) & 65(3) & 82(4) & 43(3) & -11(3) & 7(2) & -11(3) \\ \mathrm{C}(32) & 68(4) & 71(4) & 85(4) & -18(3) & 8(3) & 23(3)\end{array}$

Table 4. Hydrogen coordinates ( x 10^4) and isotropic displacement parameters $(\mathrm{A} \wedge 2$ x 10^3).

\begin{tabular}{|c|c|c|c|c|}
\hline & $\mathrm{x}$ & $\mathrm{y}$ & $\mathrm{z}$ & $\mathrm{U}(\mathrm{eq})$ \\
\hline $\mathrm{H}(3)$ & 2335 & 13296 & -2241 & 53 \\
\hline $\mathrm{H}(4)$ & 2574 & 10688 & -1863 & 58 \\
\hline $\mathrm{H}(6 \mathrm{~A})$ & 3274 & 16165 & -1200 & 71 \\
\hline $\mathrm{H}(6 \mathrm{~B})$ & 2502 & 15956 & -1588 & 71 \\
\hline $\mathrm{H}(6 \mathrm{C})$ & 3268 & 16039 & -1962 & 71 \\
\hline $\mathrm{H}(7 \mathrm{~A})$ & 3913 & 10376 & -453 & 100 \\
\hline $\mathrm{H}(7 \mathrm{~B})$ & 3674 & 9382 & -1056 & 100 \\
\hline $\mathrm{H}(7 \mathrm{C})$ & 3064 & 9863 & -552 & 100 \\
\hline $\mathrm{H}(8)$ & 4216 & 14822 & -683 & 52 \\
\hline $\mathrm{H}(9 \mathrm{~A})$ & 4422 & 12060 & -20 & 76 \\
\hline $\mathrm{H}(9 \mathrm{~B})$ & 4132 & 13581 & 276 & 76 \\
\hline $\mathrm{H}(9 \mathrm{C})$ & 4988 & 13409 & 89 & 76 \\
\hline $\mathrm{H}(12)$ & 5760 & 11548 & -2293 & 48 \\
\hline $\mathrm{H}(13)$ & 5493 & 14161 & -2653 & 49 \\
\hline $\mathrm{H}(15 \mathrm{~A})$ & 5156 & 9809 & -1256 & 77 \\
\hline $\mathrm{H}(15 \mathrm{~B})$ & 5154 & 10756 & -610 & 77 \\
\hline $\mathrm{H}(15 \mathrm{C})$ & 5915 & 10517 & -980 & 77 \\
\hline $\mathrm{H}(16 \mathrm{~A})$ & 5314 & 16710 & -1730 & 73 \\
\hline $\mathrm{H}(16 \mathrm{~B})$ & 4524 & 16452 & -1410 & 73 \\
\hline $\mathrm{H}(16 \mathrm{C})$ & 4584 & 16462 & -2171 & 73 \\
\hline H(19) & 2890 & 6478 & 2260 & 60 \\
\hline $\mathrm{H}(20)$ & 3422 & 4791 & 1446 & 62 \\
\hline $\mathrm{H}(22 \mathrm{~A})$ & 788 & 6294 & 2338 & 88 \\
\hline $\mathrm{H}(22 \mathrm{~B})$ & 1385 & 7605 & 2398 & 88 \\
\hline $\mathrm{H}(22 \mathrm{C})$ & 1450 & 6203 & 2863 & 88 \\
\hline $\mathrm{H}(23 \mathrm{~A})$ & 2691 & 3342 & 330 & 98 \\
\hline
\end{tabular}




\begin{tabular}{lrrrr}
$\mathrm{H}(23 \mathrm{~B})$ & 1998 & 2423 & 587 & 98 \\
$\mathrm{H}(23 \mathrm{C})$ & 2809 & 2199 & 907 & 98 \\
$\mathrm{H}(24)$ & 460 & 4997 & 1452 & 60 \\
$\mathrm{H}(25 \mathrm{~A})$ & 8 & 3169 & 746 & 89 \\
$\mathrm{H}(25 \mathrm{~B})$ & 524 & 2403 & 1286 & 89 \\
$\mathrm{H}(25 \mathrm{C})$ & 839 & 2669 & 587 & 89 \\
$\mathrm{H}(28)$ & 1670 & 7542 & -570 & 66 \\
$\mathrm{H}(29)$ & 1122 & 9303 & 219 & 70 \\
$\mathrm{H}(31 \mathrm{~A})$ & 1190 & 4625 & -861 & 95 \\
$\mathrm{H}(31 B)$ & 1221 & 3543 & -257 & 95 \\
$\mathrm{H}(31 \mathrm{C})$ & 1966 & 4329 & -490 & 95 \\
$\mathrm{H}(32 \mathrm{~A})$ & 572 & 8750 & 1534 & 112 \\
$\mathrm{H}(32 \mathrm{~B})$ & 29 & 7347 & 1506 & 112 \\
$\mathrm{H}(32 \mathrm{C})$ & -129 & 8731 & 1046 & 112 \\
& & & & \\
\hline
\end{tabular}

